\title{
Tailoring Highly Ordered Graphene Framework in Epoxy for High-Performance Polymer- Based Heat Dissipation Plates
}

Junfeng Ying, ${ }^{\mathrm{a}, \mathrm{b}}$ Xue Tan, ${ }^{\mathrm{a}, \mathrm{b}}$ Le Lv, ${ }^{\mathrm{a}, \mathrm{b}}$ Xiangze Wang, ${ }^{\mathrm{c}}$ Jingyao Gao, ${ }^{\mathrm{a}, \mathrm{b}}$ Qingwei Yan, ${ }^{\mathrm{a}}$ Hongbing Ma, ${ }^{\mathrm{a}}$ K. Nishimura, ${ }^{\mathrm{d}}$ He Li, ${ }^{\text {a,b }}$ Jinhong Yu, ${ }^{\text {a,b }}$ Te-Huan Liu, ${ }^{c}$ Rong Xiang, ${ }^{\text {e }}$ Rong Sun, ${ }^{\mathrm{f}}$ Nan Jiang, ${ }^{\mathrm{a}, \mathrm{b}, *}$ ChingPing Wong, ${ }^{\mathrm{g}}$ Shigeo Maruyama, ${ }^{\mathrm{e}}$ Cheng-Te Lin, ${ }^{\mathrm{a}, \mathrm{b}, *}$ and Wen Dai ${ }^{\mathrm{a}, \mathrm{b}, *}$

${ }^{a}$ Key Laboratory of Marine Materials and Related Technologies, Zhejiang Key Laboratory of Marine Materials and Protective Technologies, Ningbo Institute of Materials Technology and Engineering (NIMTE), Chinese Academy of Sciences, Ningbo 315201, P.R. China.

${ }^{b}$ Center of Materials Science and Optoelectronics Engineering, University of Chinese Academy of Sciences, Beijing 100049, P.R. China.

${ }^{c}$ School of Energy and Power Engineering, Huazhong University of Science and Technology, Wuhan, 430074, China.

d Advanced Nano-processing Engineering Lab, Mechanical Systems Engineering, Kogakuin University, Tokyo 1920015, Japan.

${ }^{e}$ Department of Mechanical Engineering, The university of Tokyo, 7-3-1 Hongo, Bunkyo-Ku, Tokyo 113-8656, Japan. Energy Nano Engineering Laboratory, National Institute of Advanced Industrial Science and Technology (AIST), Tsukuba, 305-8564, Japan.

${ }^{f}$ Shenzhen Institutes of Advanced Technology, Chinese Academy of Sciences, Shenzhen 518055, China.

${ }^{g}$ School of Materials Science and Engineering, Georgia Institute of Technology, Atlanta, Georgia 30332, United States. 


\section{This file includes:}

\section{Supplementary Figures}

Figure S1. Typical cross-sectional SEM image of the porous PU film.

Figure S2. Photographs, TGA curves, and SEM images of graphene/PU prepared using bulk PU sponge as a template.

Figure S3. Scheme and photographs of the molds using for controlling the compression ratio in the preparation process of the HOGF.

Figure S4. Characterization analysis of the MLGs used in this study.

Figure S5. Raman spectra of HOGF and CVD-grown graphene using nickel foil as the catalyst template.

Figure S6. Appearance photographs, and typical cross-sectional SEM images of the graphene framework/epoxy composites.

Figure S7. Typical cross sectional TEM images of HOGF/EP composites.

Figure S8. TGA curves of neat epoxy and HOGF/EP composites in the nitrogen atmosphere.

Figure S9. Typical SEM and TEM images of dispersed graphene/EP composite.

Figure S10. Schematic of the ANSYS simulation models and the calculated results for the HOGF/EP and dispersed graphene/EP modules.

Figure S11. Scheme illustrating the comparison of the transient heat transfer capacity along the in-plane direction of HOGF/EP, dispersed graphene/EP, and zinc.

Figure S12. Environmental temperature-dependent specific heat capacity and thermal diffusivity of neat epoxy and HOGF/EP composite.

Figure S13. Schematic of the Icepak simulation models and the calculated results with the alumina and HOGF/EP as the heat dissipation plate.

Figure S14. TMA curves and CTE of neat epoxy and graphene framework/epoxy composites.

\section{Supplementary Tables}

Table S1. The parameters for the calculation of thermal conductivities of neat epoxy and the HOGF/EP composites.

Table S2. The parameters for the calculation of thermal conductivities of dispersed graphene/EP composites.

Table S3. The key parameters for calculating the $\left\langle\cos ^{2} \theta\right\rangle$ and orientation parameter $(f)$.

Table S4. Comparison of thermal conductivities of our HOGF/EP composites with reported graphene/polymer composites.

Table S5. The detailed parameters of the components in the simulated system. 


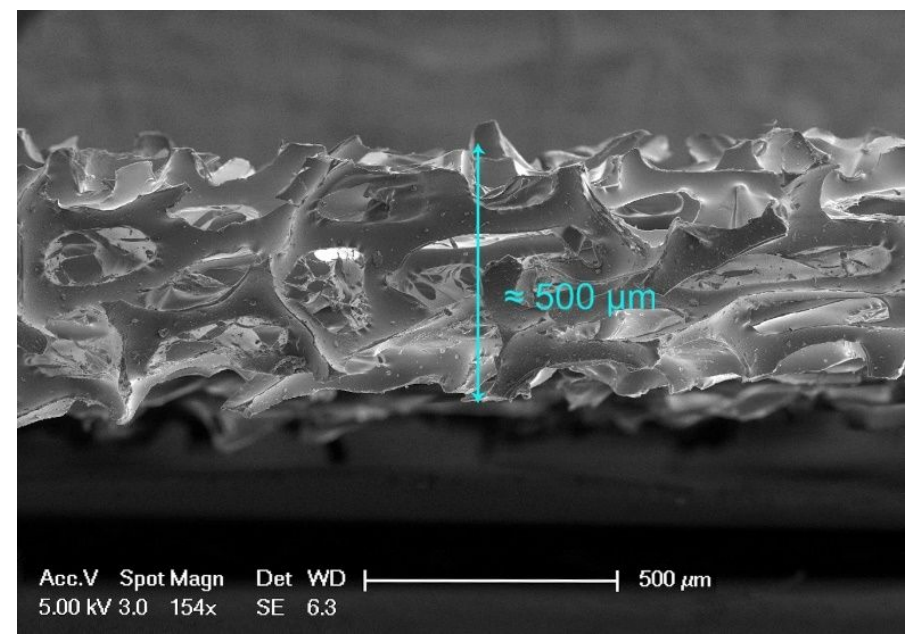

Figure S1. Typical cross-sectional SEM image of the porous PU film we used, showing an open-cell structure with an average thickness of $\approx 500 \mu \mathrm{m}$. 

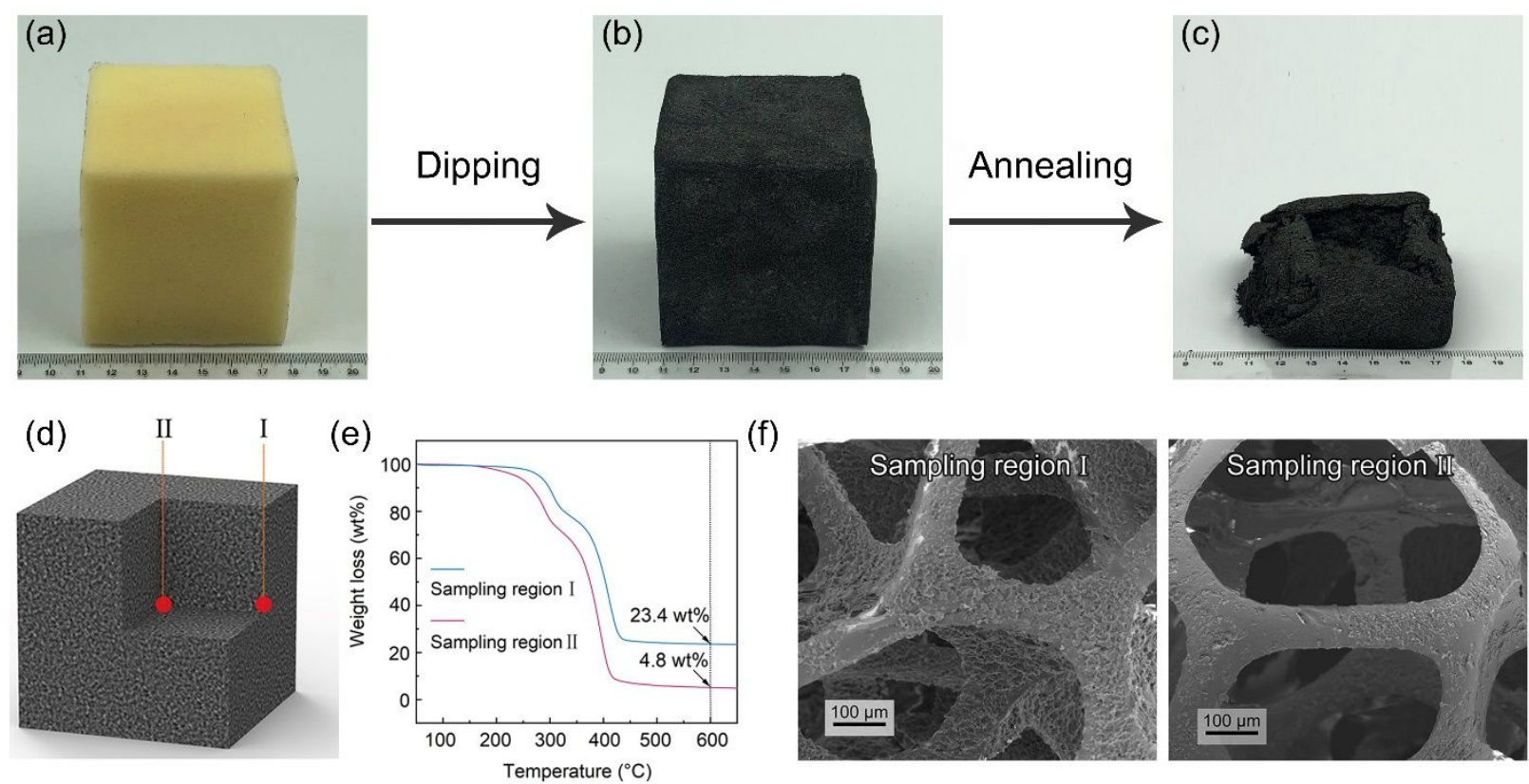

Figure S2. (a) Photograph of bulk PU sponge with a size of $7 \mathrm{~cm} \times 7 \mathrm{~cm} \times 7 \mathrm{~cm}$. Photograph of (b) the graphene/PU prepared by directly dipping the sponge bulk into the graphene dispersion (10 mg $\mathrm{mL}^{-1}$ ) with the corresponding graphene framework after thermal annealing showing in (c). (d) Schematically illustrating the sampling regions in the edge and the center of the graphene/PU. (e) TGA curves and (f) typical SEM images of the two different sampling regions.

Compared to the original dimension of the graphene/PU (Figure S2b), an apparent structural collapse of the obtained graphene framework can be found in Figure S2c, meaning that there are not enough graphene sheets inside the graphene/PU to support the structure after the PU template removal. The microscopic morphologies and graphene content in the edge and the central regions of the cubic graphene/PU monolith were investigated, with the sampling regions schematically illustrated in Figure S2d. The edge region of the monolith has a graphene content of $23.4 \mathrm{wt} \%$, while the content in the center region is as low as $4.8 \mathrm{wt} \%$ (Figure S2e and f). This result indicated that the use of a large-scale bulk PU sponge as the starting template would lead to an uneven assembly of graphene sheets. 
(a)

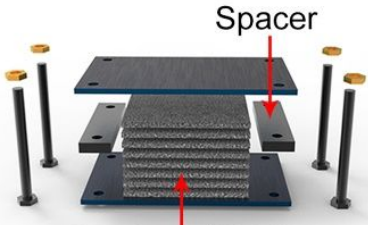

Graphene/PU film

(b)

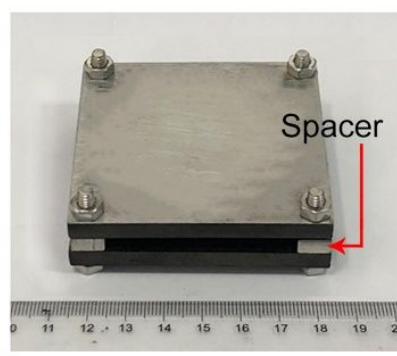

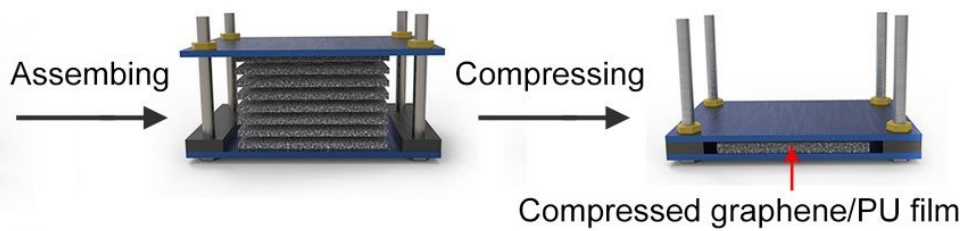

(c)

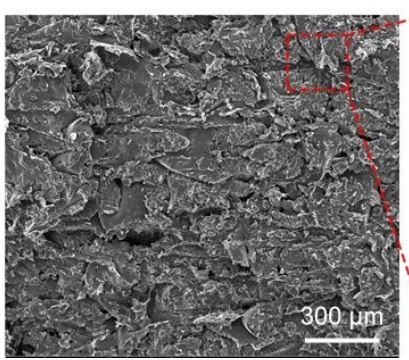

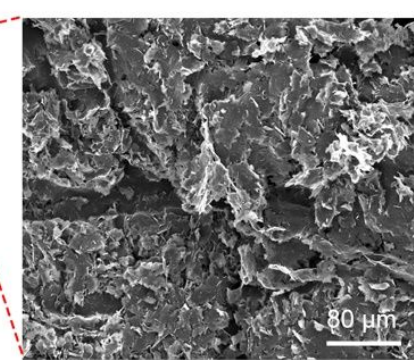

Figure S3. (a) Scheme and (b) photographs of the molds using for controlling the compression ratio in the preparation process of the HOGF. (c) Typical top view SEM images of the as-prepared HOGF.

In order to control the compression ratio, we first calculated the total thickness of the multilayerstacked composite films by multiplying the number of layers by the thickness of a single layer. For example, the total thickness of 200-layer composite films is $100 \mathrm{~mm}$ (the thickness of a single-layer film is $0.5 \mathrm{~mm}$ ). Then, to achieve a fixed compression ratio, such as controlling at 20 , we placed the $100 \mathrm{~mm}$ thick composite film in a mold with a fixed height of $5 \mathrm{~mm}$ by sandwiching a $5 \mathrm{~mm}$ thick spacer between the upper and lower plates of the mold, as shown in Figure S3a and b. After the hightemperature treatment at $800{ }^{\circ} \mathrm{C}(1 \mathrm{~h})$ to remove the PU template, we can obtain a HOGF with a thickness of $5 \mathrm{~mm}$. During the experiment, we controlled the different compression ratios by choosing spacers with different thicknesses. 
(a)
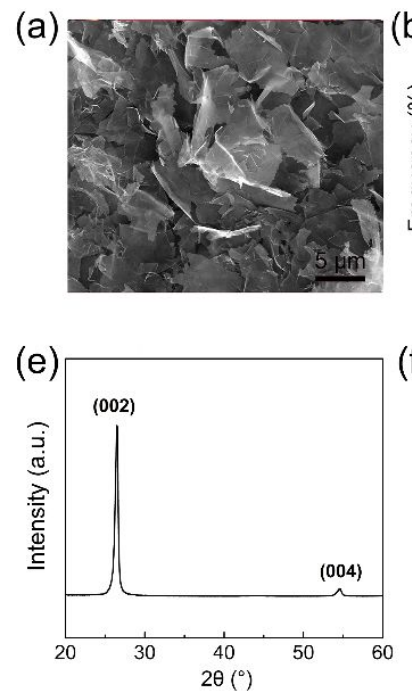

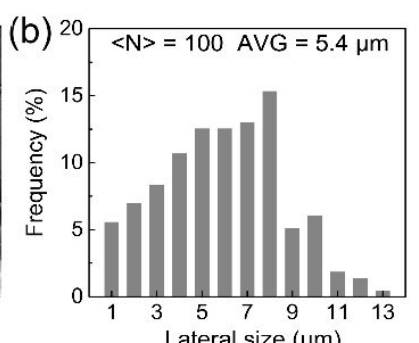

(f)

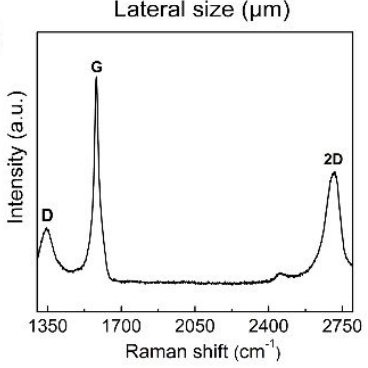

(c)

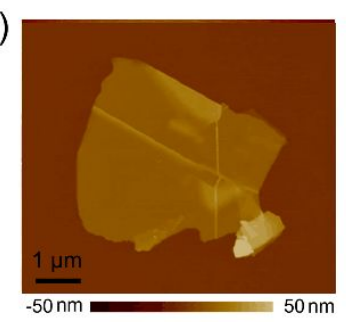

(g)

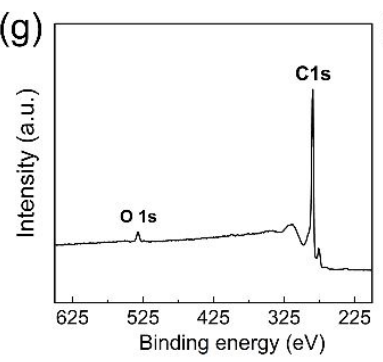

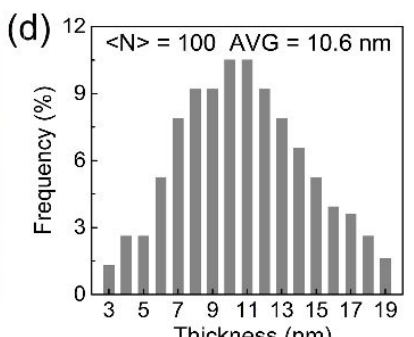

(h)

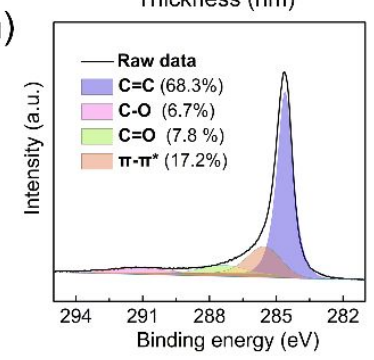

Figure S4. (a) Typical SEM image, (b) lateral size distribution, (c) AFM image, (d) thickness distribution, (e) XRD, (f) Raman, (g) XPS survey, and (h) high-resolution C1s spectra of the multilayer graphene sheets (MLGs) used in this study. The two histograms in (b) and (d) were obtained by counting one hundred graphene sheets, respectively, showing that the MLGs have an average lateral size of $5.4 \mu \mathrm{m}$ and a thickness of $10.6 \mathrm{~nm}$.

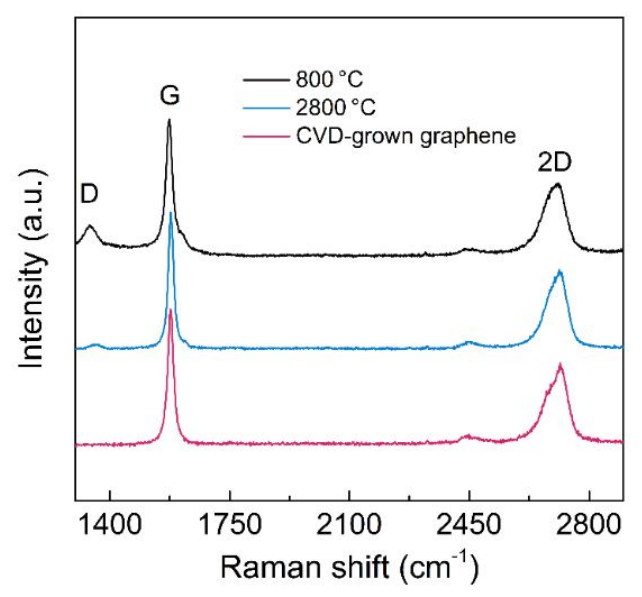

Figure S5. Raman spectra of HOGF prepared by the pyrolysis of the graphene/PU at $800{ }^{\circ} \mathrm{C}$ (black line), HOGF annealed at $2800{ }^{\circ} \mathrm{C}$ (blue line), and CVD-grown graphene using nickel (Ni) foil as the catalyst template. The comparison of the black line and the blue line shows a significant decrease of D-peak intensity by annealing the $\mathrm{HOGF}$ at $2,800{ }^{\circ} \mathrm{C}$, indicating the recovery of structural defects of graphene after graphitization post-treatment. 
(a)
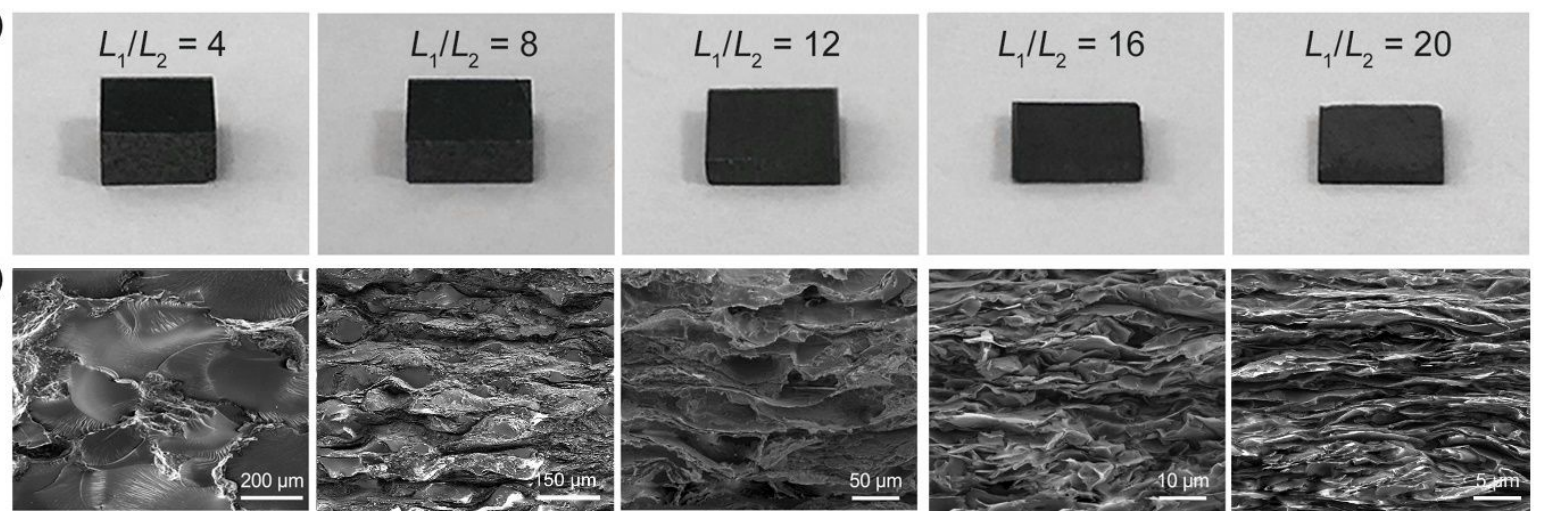

Figure S6. (a) Appearance photographs, and (b) typical cross-sectional SEM images of the graphene framework/epoxy composites prepared by performing different compression ratio $\left(L_{1} / L_{2}\right)$ to the multilayer-stacked composite films.
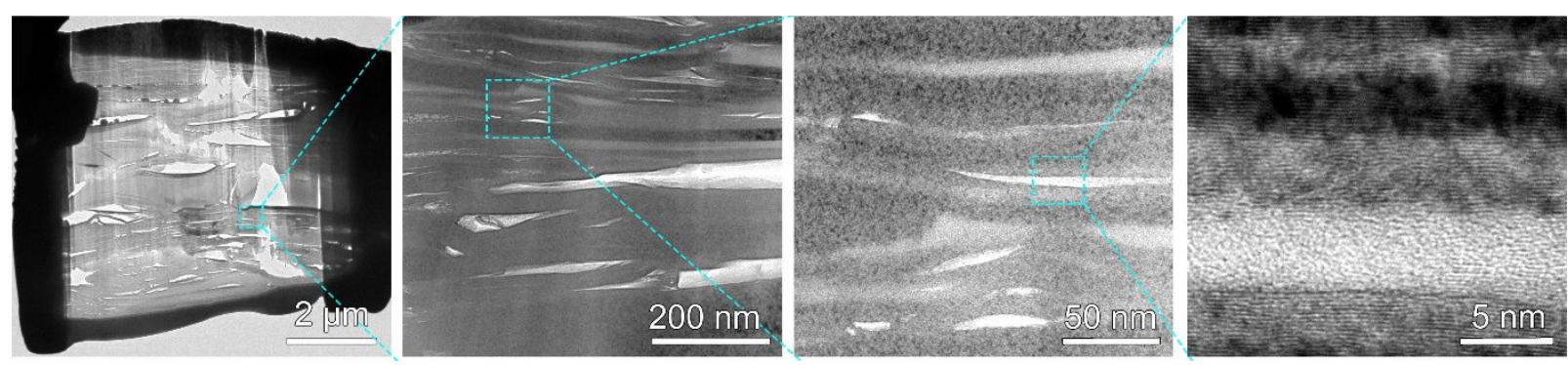

Figure S7. Typical cross sectional TEM images of HOGF/EP composites with different resolutions. 


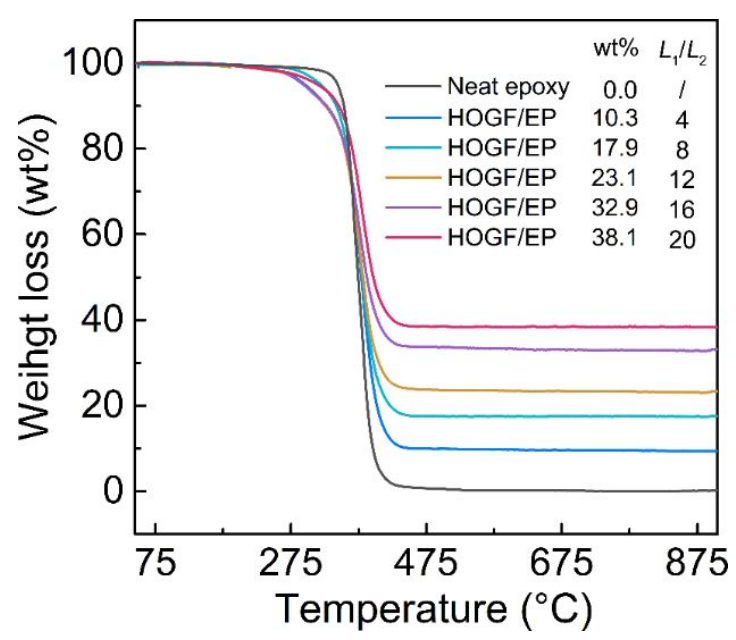

Figure S8. TGA curves of neat epoxy and HOGF/EP composites in the nitrogen atmosphere. The volume fraction of graphene $\left(V_{\mathrm{G}}\right)$ can be calculated using the equation of $V_{\mathrm{G}}=\frac{\omega_{\mathrm{G}} / \rho_{\mathrm{G}}}{1 / \rho_{\text {composite }}}$, where $\omega_{\mathrm{G}}$ is the mass fraction of graphene showing in Figure S8, $\rho_{\mathrm{G}}$ and $\rho_{\text {composite }}$ are the density of graphene $\left(2.1 \mathrm{~g} \mathrm{~cm}^{-3}\right)$ and the HOGF/EP composite (Table S1), respectively. 
Table S1. The parameters for the calculation of in-plane $(\kappa / /)$ and through-plane $(\kappa / /)$ thermal conductivities of neat epoxy and the HOGF/EP composites. The specific heat capacity $\left(C_{\mathrm{p}}\right)$ of all samples was evaluated using a differential scanning calorimeter (DSC) analysis.

\begin{tabular}{|c|c|c|c|c|c|c|c|}
\hline $\begin{array}{c}\text { Compressio } \\
\text { n ratio } \\
\left(L_{1} / L_{2}\right)\end{array}$ & $\begin{array}{r}\text { Gra } \\
\text { cor } \\
\text { (vol } \%\end{array}$ & $\begin{array}{l}\text { hene } \\
\text { ent } \\
w t \%)\end{array}$ & $\begin{array}{l}\text { Thermal } \\
\text { diffusivity } \\
\left(\mathrm{mm}^{2} \mathrm{~s}^{-1}\right)\end{array}$ & $\begin{array}{l}\text { Specific } \\
\text { heat } \\
\text { capacity } \\
\left(\mathrm{J} \mathrm{g}^{-1} \mathrm{~K}^{-1}\right)\end{array}$ & $\begin{array}{l}\text { Density } \\
\left(\mathrm{g} \mathrm{cm}^{-3}\right)\end{array}$ & $\begin{array}{l}\text { Thermal } \\
\text { conductivity } \\
\left(\mathrm{W} \mathrm{m}^{-1} \mathrm{~K}^{-1}\right)\end{array}$ & Direction \\
\hline l & 0 & 0 & $0.12 \pm 0.01$ & $\approx 1.34$ & $1.189 \pm 0.012$ & $0.19 \pm 0.01$ & Isotropic \\
\hline \multirow{2}{*}{4} & \multirow{2}{*}{6.2} & \multirow{2}{*}{10.3} & $8.20 \pm 1.12$ & \multirow{2}{*}{$\approx 1.28$} & \multirow{2}{*}{$1.235 \pm 0.027$} & $12.96 \pm 1.84$ & $\kappa_{/ /}$ \\
\hline & & & $3.41 \pm 0.27$ & & & $5.39 \pm 0.45$ & $\kappa_{\perp}$ \\
\hline \multirow{2}{*}{8} & \multirow{2}{*}{10.6} & \multirow{2}{*}{17.9} & $20.04 \pm 2.86$ & \multirow{2}{*}{$\approx 1.23$} & \multirow{2}{*}{$1.243 \pm 0.032$} & $30.64 \pm 4.43$ & $\kappa_{/ /}$ \\
\hline & & & $4.61 \pm 0.59$ & & & $7.05 \pm 0.93$ & $\kappa_{\perp}$ \\
\hline \multirow{2}{*}{12} & \multirow{2}{*}{13.9} & \multirow{2}{*}{23.1} & $31.73 \pm 1.92$ & \multirow{2}{*}{$\approx 1.20$} & \multirow{2}{*}{$1.260 \pm 0.038$} & $47.98 \pm 2.87$ & $\kappa_{/ /}$ \\
\hline & & & $5.89 \pm 0.64$ & & & $8.91 \pm 0.98$ & $\kappa_{\perp}$ \\
\hline \multirow{2}{*}{16} & \multirow{2}{*}{20.8} & \multirow{2}{*}{32.9} & $56.96 \pm 2.58$ & \multirow{2}{*}{$\approx 1.14$} & \multirow{2}{*}{$1.327 \pm 0.042$} & $86.17 \pm 3.78$ & $\kappa_{/ /}$ \\
\hline & & & $7.38 \pm 0.28$ & & & $11.16 \pm 0.47$ & $\kappa_{\perp}$ \\
\hline \multirow{2}{*}{20} & \multirow{2}{*}{24.7} & \multirow{2}{*}{38.1} & $78.42 \pm 2.05$ & \multirow{2}{*}{$\approx 1.10$} & \multirow{2}{*}{$1.362 \pm 0.053$} & $117.49 \pm 3.15$ & $\kappa_{/ /}$ \\
\hline & & & $8.94 \pm 0.23$ & & & $13.39 \pm 0.43$ & $\kappa_{\perp}$ \\
\hline
\end{tabular}



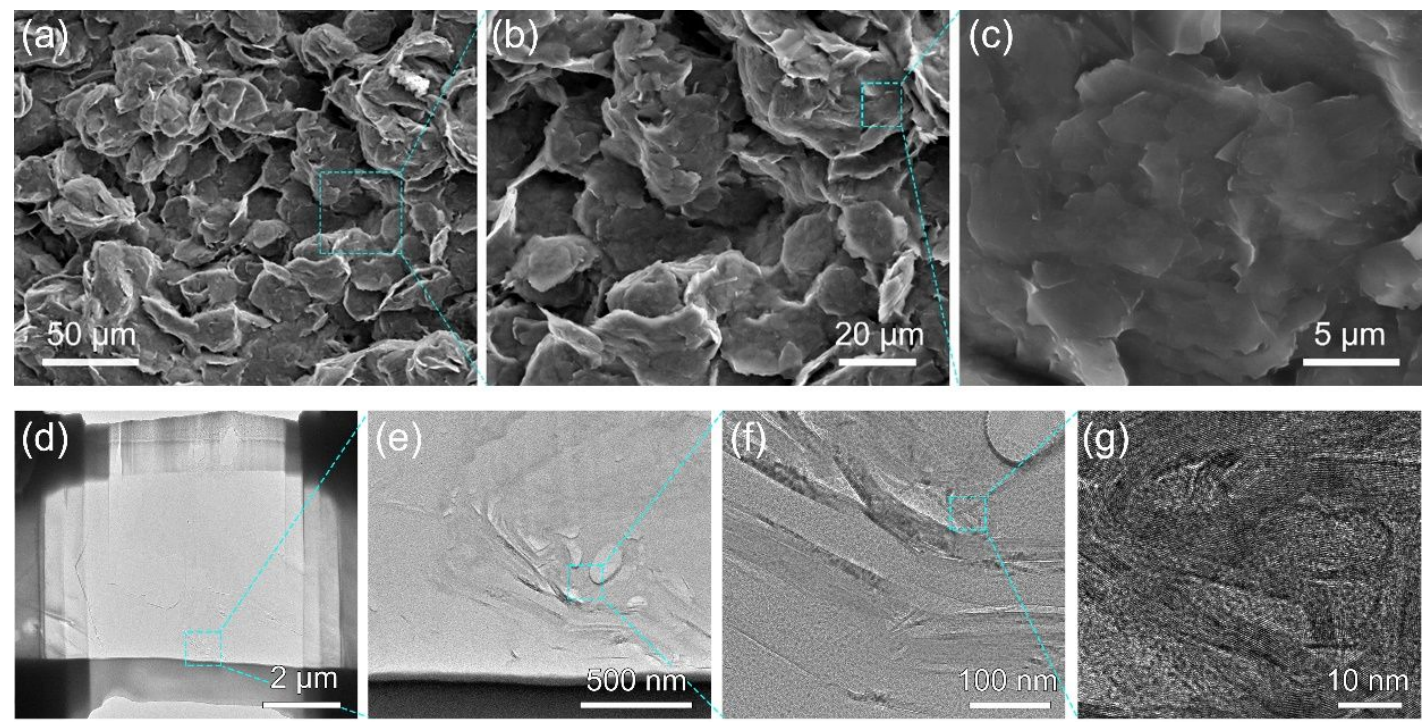

Figure S9. (a-c) Typical SEM and (d-g) TEM images of dispersed graphene/EP composite, showing the random distribution of graphene sheets in epoxy matrix.

Table S2. The parameters for the calculation of thermal conductivities of dispersed graphene/EP composites.

\begin{tabular}{cccccc}
\hline $\begin{array}{c}\text { Graphene } \\
\text { content } \\
\text { (vol\% } / \mathrm{wt} \%)\end{array}$ & $\begin{array}{c}\text { Thermal } \\
\text { diffusivity } \\
\left(\mathrm{mm}^{2} \mathrm{~s}^{-1}\right)\end{array}$ & $\begin{array}{c}\text { Specific heat } \\
\text { capacity } \\
\left(\mathrm{J} \mathrm{g}^{-1} \mathrm{~K}^{-1}\right)\end{array}$ & $\begin{array}{c}\text { Density } \\
\left(\mathrm{g} \mathrm{cm}^{-3}\right)\end{array}$ & $\begin{array}{c}\text { Thermal } \\
\text { conductivity } \\
\left(\mathrm{W} \mathrm{m}^{-1} \mathrm{~K}^{-1}\right)\end{array}$ \\
\hline 6.2 & 10.3 & $1.24 \pm 0.09$ & $\approx 1.28$ & $1.265 \pm 0.016$ & $2.00 \pm 0.11$ \\
\hline 11.3 & 17.9 & $1.59 \pm 0.07$ & $\approx 1.23$ & $1.322 \pm 0.023$ & $2.59 \pm 0.09$ \\
\hline 14.6 & 23.1 & $3.11 \pm 0.23$ & $\approx 1.20$ & $1.327 \pm 0.036$ & $4.95 \pm 0.26$ \\
\hline 20.2 & 32.9 & $3.73 \pm 0.39$ & $\approx 1.14$ & $1.288 \pm 0.057$ & $5.48 \pm 0.45$ \\
\hline 23.1 & 38.1 & $5.15 \pm 0.58$ & $\approx 1.10$ & $1.273 \pm 0.078$ & $7.21 \pm 0.66$ \\
\hline
\end{tabular}




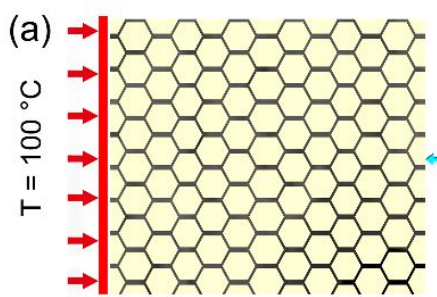

HOGF/EP

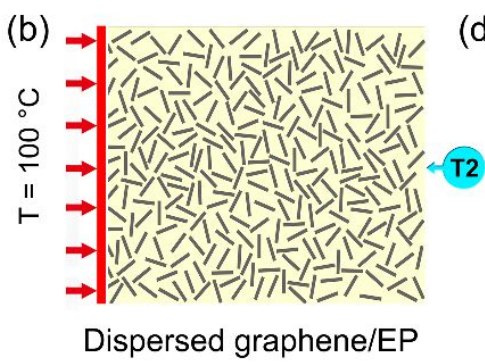

(c)
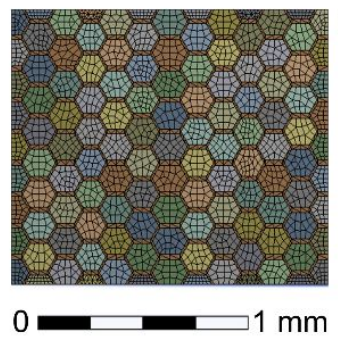

(d)

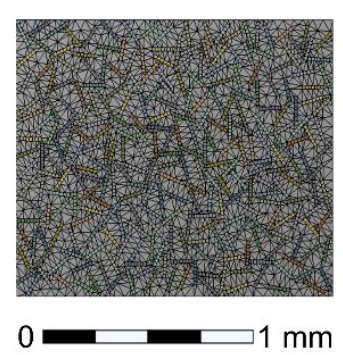

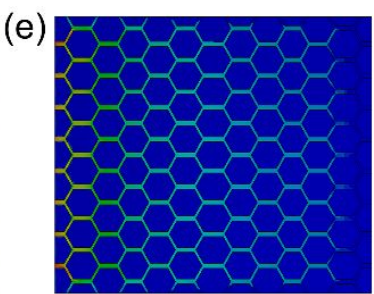

0 대 $1.3 e 8 \mathrm{~W} \mathrm{~m}^{-2}$

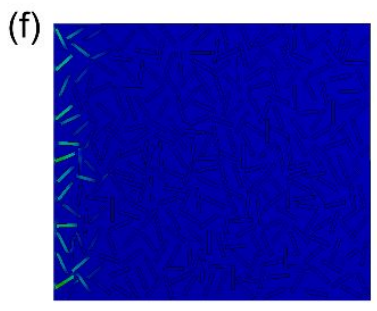

0
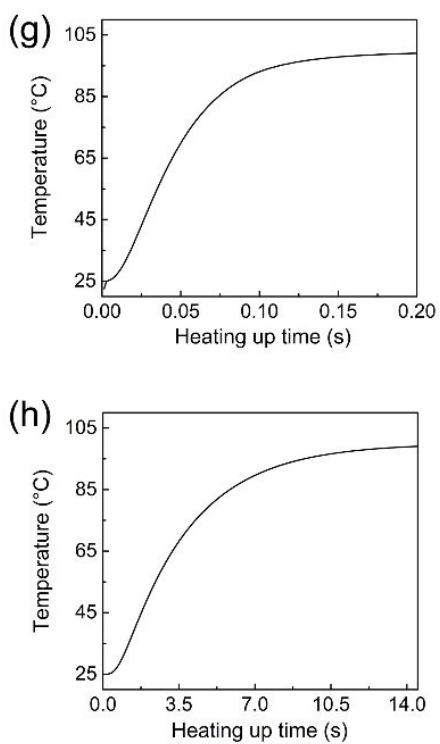

Figure S10. Schematic of the ANSYS simulation models for the (a) HOGF/EP and (b) dispersed graphene/EP modules, with the corresponding grid division showing in (c) and (d), respectively. Simulated transient heat flux profiles for (e) the HOGF/EP and (f) dispersed graphene/EP modules. The temperature evolution of the measured points versus the heating up time for $(\mathrm{g})$ the HOGF/EP and (h) dispersed graphene/EP modules.

The transient thermal response and heat flux distribution of the HOGF/EP and dispersed graphene/EP during heating were simulated using commercial computational fluid dynamics software (ANSYS) with the corresponding simulation models shown in Figure S10a and b. In Figure S10a, the graphene sheets composing the HOGF had formed a continuous and interconnected framework structure (the black region, 24.7 vol\%). Therefore, in order to simplify the ANSYS simulation model, we took the foam skeleton as the research object and set the thermal conductivity of an individual graphene skeleton to $560 \mathrm{~W} \mathrm{~m}^{-1} \mathrm{~K}^{-1}$. In Figure S10b, the black region is the dispersed graphene sheets, which are discretely distributed in the polymer matrix. Therefore, we took an individual sheet takes the foam skeleton as the research object and set the thermal conductivity of $1900 \mathrm{~W} \mathrm{~m}^{-1} \mathrm{~K}^{-1}$, which is the basal plane thermal conductivity of the multilayer graphene sheets (MLGs) after undergoing a graphitization post-treatment. The yellow regions in Figure S10a and b both refer to epoxy, with the 
thermal conductivity of $0.19 \mathrm{~W} \mathrm{~m}^{-1} \mathrm{~K}^{-1}$.

The computational domain of the simulation models for the two cases is set to $1.5 \mathrm{~mm} \times 1.3 \mathrm{~mm}$ $\times 0.2 \mathrm{~mm}$, with the corresponding grid division showing in Figure S10c and d, respectively. The initial conditions of the two models are set to $25^{\circ} \mathrm{C}$, and adiabatic boundary conditions are specified at the starting state. Then, two constant heat sources with a temperature of $100^{\circ} \mathrm{C}$ (schematically illustrated as the red line) were applied at the left sides of the HOGF/EP and dispersed graphene/EP modules, respectively, leading to the formation of one-dimensional heat conduction through the two modules. When the transient analysis started, we set two temperature probes (T1 and T2) located on the right side of the two modules to measure the transient thermal response curves. The heat flux distribution of the HOGF/EP and dispersed graphene/EP modules at the starting point $(\mathrm{t}=0 \mathrm{~s})$ were presented in Figure S10e and f, respectively, in which maximal heat flux of the HOGF/EP modules is over two orders of magnitude higher than that of dispersed graphene/EP modules. And the heat flow in HOGF/EP modules is mainly distributed along with the graphene skeleton, indicating that the HOGF severed as the continuous heat channels in the epoxy play a pivotal role in achieving a fast thermal response rate. In Figure S10g and h, it just takes 0.2 for the HOGF/EP module to reach a near steadystate $\left(\mathrm{T} 1=\mathrm{T} 2=99{ }^{\circ} \mathrm{C}\right)$, whereas the time need for the dispersed graphene/EP case is as long as 14.5 s, demonstrating a superior heat transfer rate of the interconnected HOGF. 
(a)

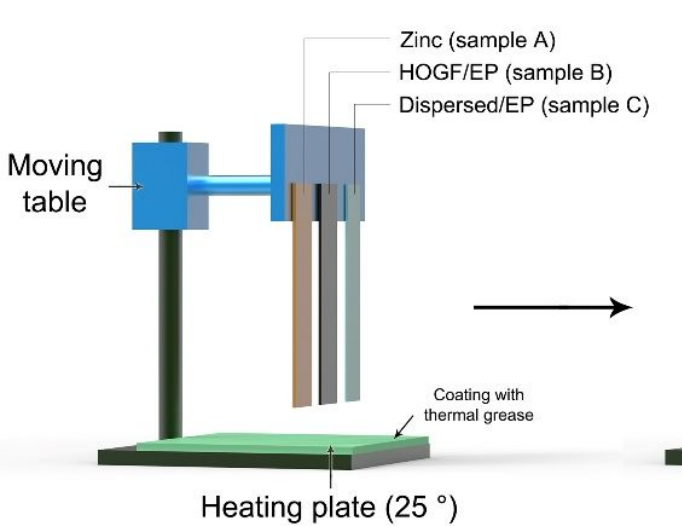

Heating plate $\left(25^{\circ}\right)$ (b)

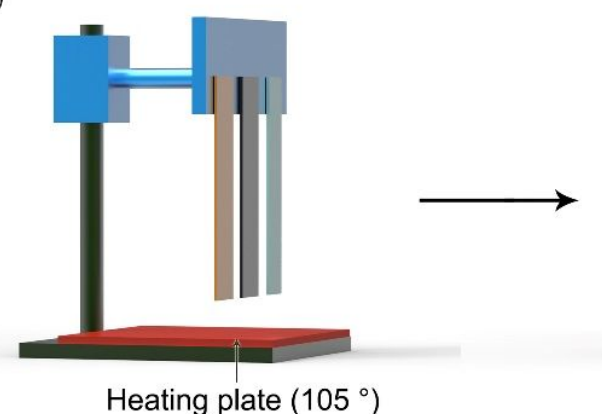

Heating plate $\left(105^{\circ}\right)$ (c)

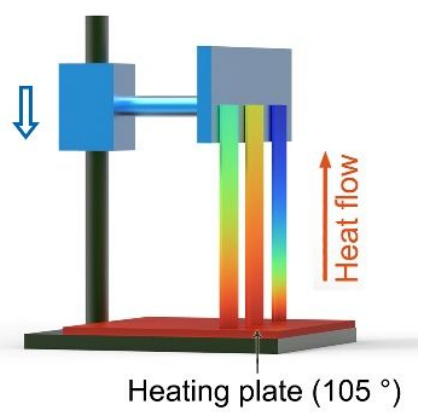

Figure S11. Scheme illustrating the comparison of the transient heat transfer capacity along the inplane direction of HOGF/EP, dispersed graphene/EP, and zinc through an experimental approach. (a) Test system configuration. Three samples were attached to the vertical moving table and not in contact with the heating plate. (b) Test preparation. The heating plate was heated to a steady-state temperature of $105{ }^{\circ} \mathrm{C}$. (c) Test starting. Move the vertical moving table down so that the three samples were in contact with the heating plate, which was coated with thermal grease to ensure the same contact state of the three samples. In this process, the real-time temperature evolution of the three samples was recorded using a calibrated infrared (IR) camera. 
Table S3. The key parameters for calculating the $\left\langle\cos ^{2} \theta\right\rangle$ and orientation parameter $(f)$. $V_{\mathrm{G}}$ was obtained based on the TGA analysis shown in Figure S8. $V_{\text {air }}$ is the volume fraction of the existing air bubbles in the composites, and can be calculated using the equation of $V_{\text {air }}=1-\rho_{\text {composite }} / \rho_{\text {theoretical }}$, where $\rho_{\text {composite }}$ and $\rho_{\text {theoretical }}$ are the measured and theoretical density of the graphene framework/epoxy composites, respectively. $V_{\mathrm{EP}}$ is obtained based on the equation of $V_{\mathrm{EP}}=1-V_{\mathrm{G}}-V_{\mathrm{Air}}$.

\begin{tabular}{ccccccccc}
\hline $\begin{array}{c}\text { Graphene } \\
\text { content (vol\%) }\end{array}$ & $\begin{array}{c}\rho_{\text {composite }} \\
\left(\mathrm{g} \mathrm{cm}^{-3}\right)\end{array}$ & $\begin{array}{c}\rho_{\text {theoretical }} \\
\left(\mathrm{g} \mathrm{cm}^{-3}\right)\end{array}$ & $V_{\mathrm{G}}$ & $V_{\text {air }}$ & $V_{\mathrm{EP}}$ & $\begin{array}{c}\kappa_{\text {strut }} \\
\left(\mathrm{W} \mathrm{m}^{-1} \mathrm{~K}^{-1}\right)\end{array}$ & $\begin{array}{c}\kappa_{\text {air }} \\
\left(\mathrm{W} \mathrm{m}^{-1} \mathrm{~K}^{-1}\right)\end{array}$ & $\begin{array}{c}\kappa_{\mathrm{EP}} \\
\left(\mathrm{W} \mathrm{m}^{-1} \mathrm{~K}^{-1}\right)\end{array}$ \\
\hline 6.2 & 1.235 & 1.283 & 0.062 & 0.037 & 0.901 & & & \\
10.6 & 1.243 & 1.352 & 0.106 & 0.081 & 0.813 & & & \\
13.9 & 1.260 & 1.399 & 0.139 & 0.099 & 0.762 & 560 & 0.026 & 0.19 \\
\hline 20.8 & 1.327 & 1.489 & 0.208 & 0.109 & 0.683 & & & \\
\hline 24.7 & 1.362 & 1.536 & 0.247 & 0.113 & 0.640 & & & \\
\hline
\end{tabular}


Table S4. Comparison of thermal conductivities of our HOGF/EP composites with reported graphene/polymer composites.

\begin{tabular}{|c|c|c|c|c|c|c|}
\hline Filler & Matrix & $\begin{array}{c}\text { Thermal } \\
\text { Conductivity } \\
\left(\mathrm{W} \mathrm{m}^{-1} \mathrm{~K}^{-1}\right)\end{array}$ & $\begin{array}{c}\text { Graphene } \\
\text { content } \\
(\mathrm{vol} \%)\end{array}$ & $\begin{array}{l}\text { TCE } \\
(\%)\end{array}$ & Direction & Ref. \\
\hline \multicolumn{7}{|l|}{ Randomly dispersed graphene sheets as filler } \\
\hline Graphene and copper nanoparticle & Epoxy & 6.9 & 27.4 & 3532 & Isotropic & S2 \\
\hline Multilayer graphene & Epoxy & 1.5 & 2.8 & 683 & Isotropic & $\mathrm{S} 3$ \\
\hline Graphene nanoplatelet & Epoxy & 12.4 & 24.11 & 6100 & Isotropic & S4 \\
\hline Multilayer graphene & Epoxy & 5.1 & 10 & 2300 & Isotropic & S5 \\
\hline Graphite Nanoplatelet/carbon nanotube & Epoxy & 1.75 & 5.98 & 775 & Isotropic & S6 \\
\hline Exfoliated graphite & Epoxy & 5.8 & 12.4 & 2800 & Isotropic & S7 \\
\hline Graphene nanoplatelets & Polycarbonate & 7.3 & 9.1 & 2942 & Isotropic & S8 \\
\hline \multicolumn{7}{|l|}{ Isotropic graphene-based framework as filler } \\
\hline High-quality graphene aerogel & Octadecanol & 4.28 & 2 & 1800 & Isotropic & S9 \\
\hline Hybrid graphene aerogel & PEG & 1.43 & 1 & 361 & Isotropic & $\mathrm{S} 10$ \\
\hline High-density graphene hybrid aerogel & Octadecanol & 5.92 & 5.5 & 2600 & Isotropic & $\mathrm{S} 11$ \\
\hline Ultrathin graphite foams/carbon nanotube & Erythritol & 4.1 & 1.25 & 425 & Isotropic & $\mathrm{S} 12$ \\
\hline Graphene- silicon carbide nanowire & Polyimide & 2.63 & 6.54 & 989 & Isotropic & $\mathrm{S} 13$ \\
\hline Ultrathin graphite foam & Paraffin & 2.8 & 1.2 & 1800 & Isotropic & $\mathrm{S} 14$ \\
\hline \multicolumn{7}{|l|}{ Anisotropic graphene-based framework as filler } \\
\hline Lamellar-structured graphene aerogel & Epoxy & 20 & 2.3 & 9915 & $\kappa_{/ /}$ & $\mathrm{S} 15$ \\
\hline Graphene aerogels with highly aligned network & Epoxy & 6.57 & 0.75 & 3700 & $\kappa_{\perp}$ & $\mathrm{S} 16$ \\
\hline Vertically aligned graphene network & Epoxy & 2.13 & 0.92 & 1213 & $\kappa_{\perp}$ & S17 \\
\hline Aligned carbon nanotube & Epoxy & 4.87 & 16.7 & 2335 & $\kappa_{\perp}$ & $\mathrm{S} 18$ \\
\hline Reduced graphene oxide/boron nitride aerogel & Epoxy & 11.01 & 25.4 & 5405 & $\kappa_{\perp}$ & S19 \\
\hline Quasi-isotropic graphene framework & Epoxy & 10 & 3.19 & 4900 & $\kappa_{/ /}$ & $\mathrm{S} 20$ \\
\hline Vertically aligned graphene foam & Epoxy & 35.5 & 19 & 17650 & $\kappa_{\perp}$ & $\mathrm{S} 21$ \\
\hline Graphene nanoflake & PTFE & 10 & 25 & 5000 & $\kappa_{\perp}$ & $\mathrm{S} 22$ \\
\hline Aligned multilayer graphene & Epoxy & 16.75 & 7.04 & 8275 & $\kappa_{/ /}$ & $\mathrm{S} 23$ \\
\hline Graphene woven fabric & Polyimide & 3.73 & 7.7 & 1418 & $\kappa_{/ /}$ & $\mathrm{S} 1$ \\
\hline Arranged Ni-template graphene foam & Natural rubber & 10.64 & 6.2 & 8100 & $\kappa_{/ /}$ & S24 \\
\hline Ni templated 3D graphene & Epoxy & 8.8 & 4.87 & 4300 & $\kappa_{/ /}$ & $\mathrm{S} 25$ \\
\hline
\end{tabular}

This work

Highly ordered graphene framework (HOGF)

Epoxy

117

$24.7 \quad 61600$

$\kappa / /$ 

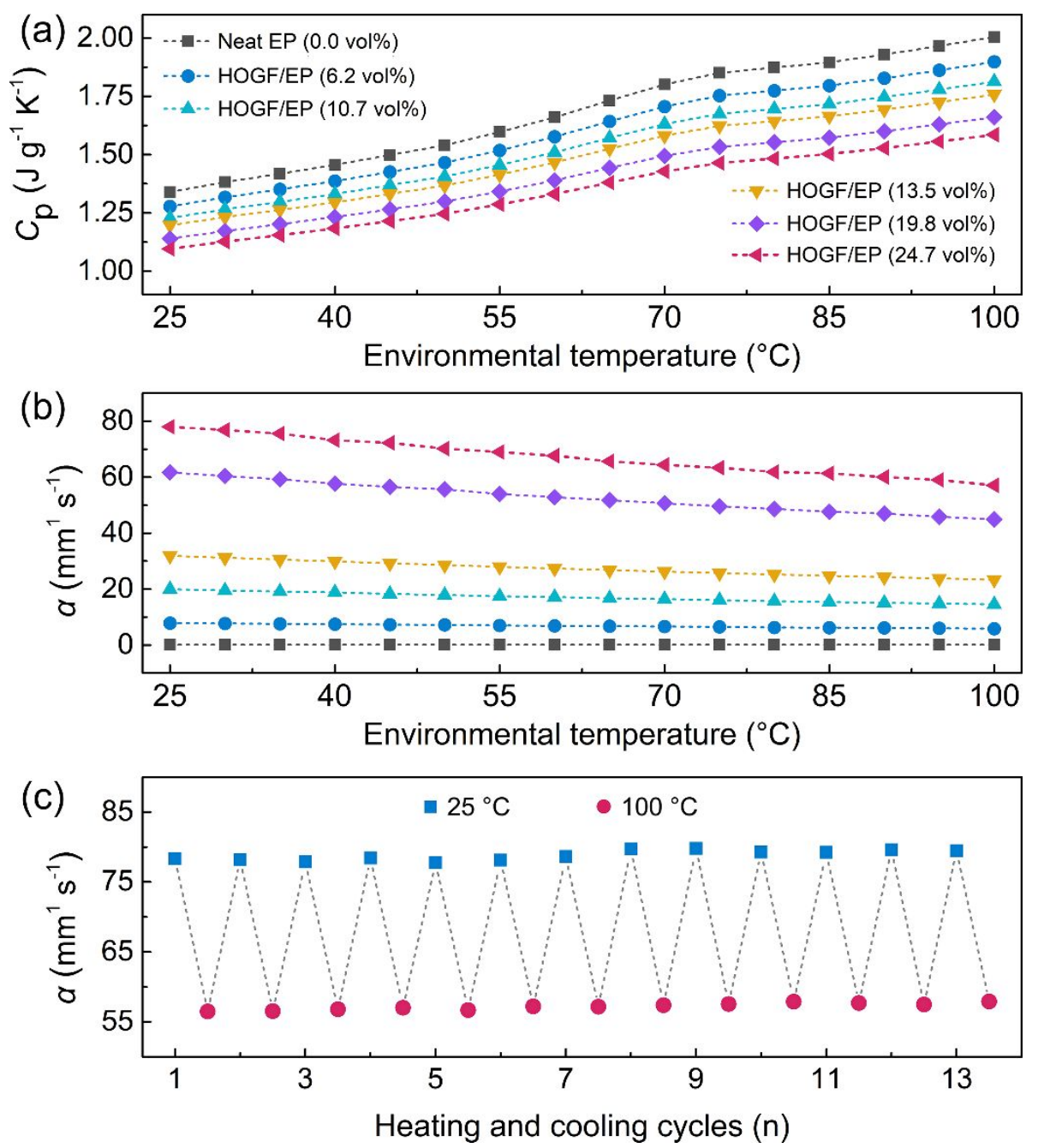

Figure S12. Environmental temperature-dependent (a) specific heat capacity $\left(C_{\mathrm{p}}\right)$ and (b) thermal diffusivity $(\alpha)$ of neat epoxy and HOGF/EP composite. (c) The variational $\alpha$ of HOGF/EP (24.7 vol\%) during the cyclic heating/cooling test. 
(a)

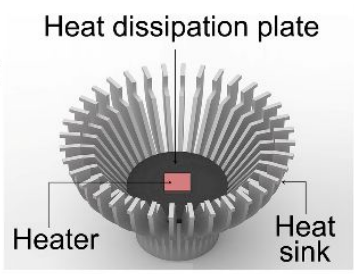

Schematic of cooling system (b)

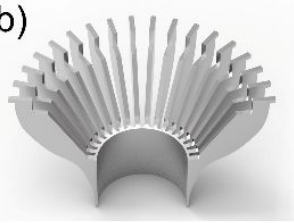

Before integration

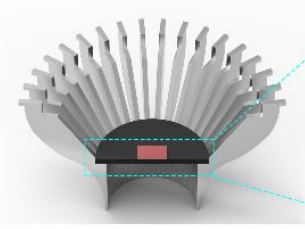

After integration (c)

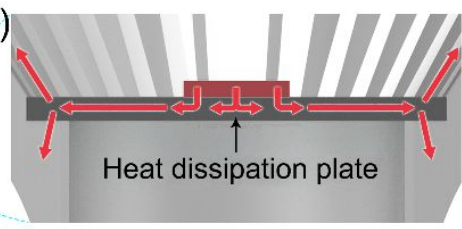

Heat flow pathway $\rightarrow$ (d)

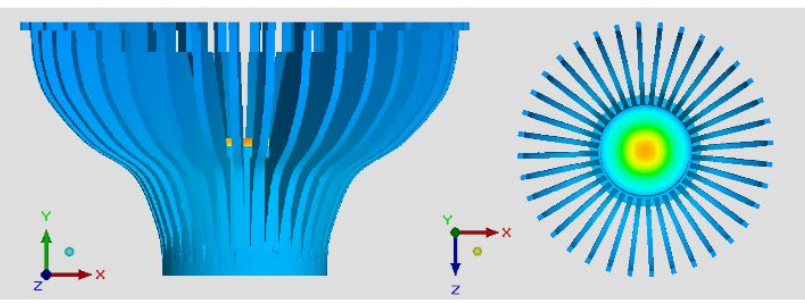

(f)

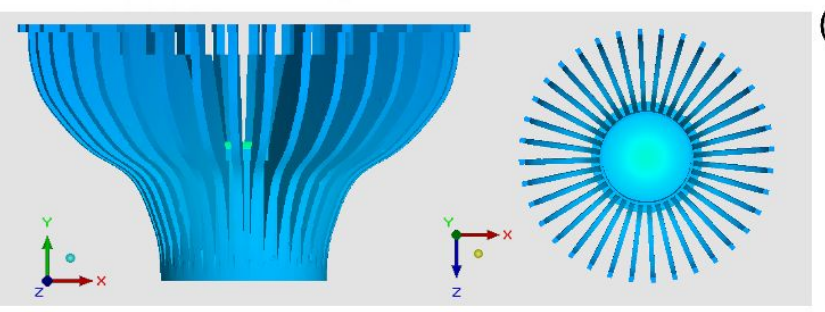

(e)

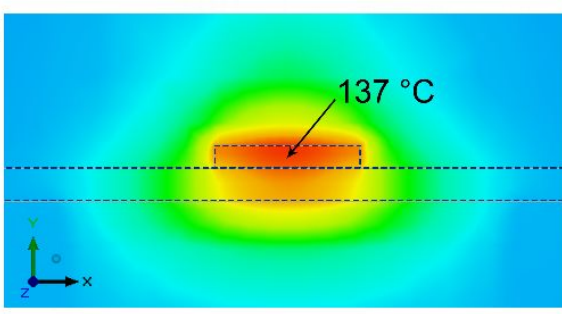

(g)

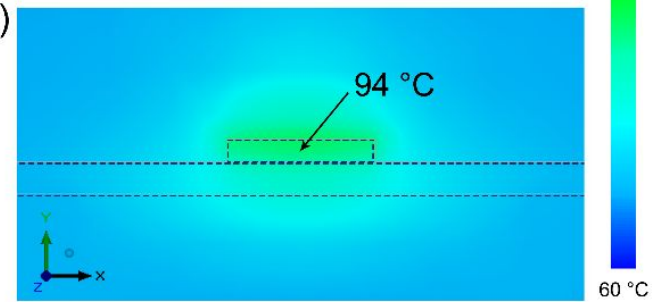

Figure S13. (a) Icepak system model for evaluating the thermal management performance of the heat dissipation plate. (b) The integration of the dissipation plate on a sunflower heat sink for cooling the LED chip with the heat flow pathway showing in (c). (d) Simulated overall temperature cloud map and (e) cross-sectional temperature distribution of the cooling system using alumina as the heat dissipation plate. (d) Simulated overall temperature cloud map and (e) cross-sectional temperature distribution of the cooling system using HOGF/EP as the heat dissipation plate.

In order to further understand the role of the heat dissipation plate, we used commercial computational fluid dynamics software (Icepak) to simulate the heat transfer process of the cooling system. The model implementation was shown in Figure S13a-c, in which the power density of the heater (LED chip) was set to $10 \mathrm{~W} \mathrm{~cm}^{-2}$, and the background temperature of the whole system was set to $25^{\circ} \mathrm{C}$ at $1 \mathrm{~atm}$ in the atmosphere. The detailed parameters of the heater, heat sink, and the two heat dissipation plates were listed in Table S5. Figure S13d-g presented the simulated overall temperature 
cloud map and cross-sectional temperature distribution of the cooling system, demonstrating a better heat dissipation capability of our HOGF/EP compared to that of alumina as the heat dissipation.

Table S5. The detailed parameters of the components in the simulated system.

\begin{tabular}{lcccc}
\hline & Size $\left(\mathrm{cm}^{3}\right)$ & Materials & $\kappa\left(\mathrm{W} \mathrm{m}^{-1} \mathrm{~K}^{-1}\right)$ & $C_{\mathrm{p}}\left(\mathrm{J} \mathrm{g}^{-1} \mathrm{~K}^{-1}\right)$ \\
\hline Heater & $1 \times 1 \times 0.1$ & Alumina & 27 & 0.91 \\
Heat sink & Sunflower & Aluminum & 237 & 0.88 \\
Heat dissipation plate \#1 & $\Phi 4.0 \times 0.2$ & Alumina & 27 & 0.91 \\
& & & $117(\mathrm{X}-\mathrm{Z})$ & 1.10 \\
Heat dissipation plate \#2 & $\Phi 4.0 \times 0.2$ & HOGF/EP & $13.4(\mathrm{Y})$ & \\
\hline
\end{tabular}

(a)

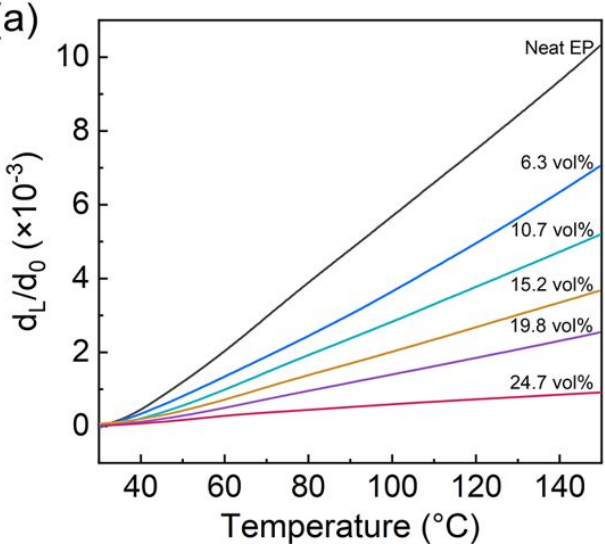

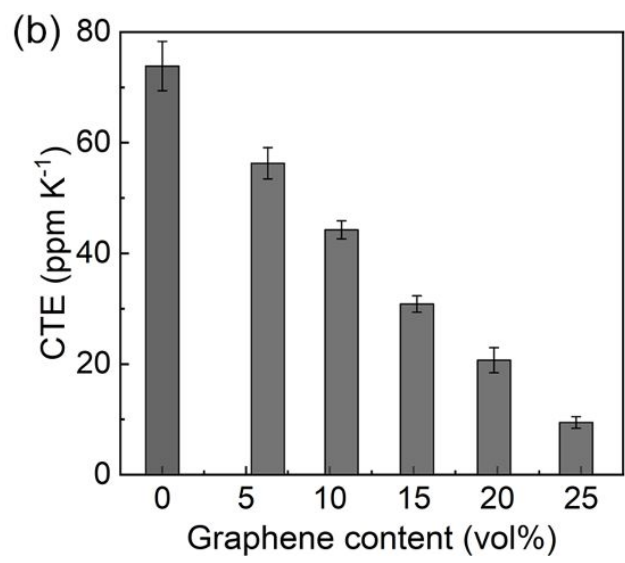

Figure S14. (a) TMA curves of neat epoxy and graphene framework/epoxy composites as a function of testing temperature. (b) The calculated average coefficient of thermal expansion (CTE) of neat epoxy and graphene framework/epoxy composites between 40 to $140{ }^{\circ} \mathrm{C}$. The $\mathrm{CTE}$ of these composites decreases with the increase of the graphene addition in the epoxy, and the lowest value of $9.4 \mathrm{ppm} \mathrm{K}^{-1}$ can be obtained at the graphene content of $24.7 \mathrm{vol} \%$. 


\section{REFERENCES}

(S1) Gong, J.; Liu, Z.; Yu, J.; Dai, D.; Dai, W.; Du, S.; Li, C.; Jiang, N.; Zhan, Z.; Lin, C. T., Graphene Woven Fabric-Reinforced Polyimide Films with EnhancFanded and Anisotropic Thermal Conductivity. Composites, Part A 2016, 87, 290-296.

(S2) Barani, Z.; Mohammadzadeh, A.; Geremew, A.; Huang, C. Y.; Coleman, D.; Mangolini, L.; Kargar, F.; Balandin, A. A., Thermal Properties of the Binary-Filler Hybrid Composites with Graphene and Copper Nanoparticles. Adv. Funct. Mater. 2020, 30, 1904008.

(S3) Shen, X.; Wang, Z.; Wu, Y.; Liu, X.; He, Y. B.; Kim, J. K., Multilayer Graphene Enables Higher Efficiency in Improving Thermal Conductivities of Graphene/Epoxy Composites. Nano Lett. 2016, 16, 3585-3593.

(S4) Shtein, M.; Nadiv, R.; Buzaglo, M.; Kahil, K.; Regev, O., Thermally Conductive GraphenePolymer Composites: Size, Percolation, and Synergy Effects. Chem. Mater. 2015, 27, 2100-2106.

(S5) Shahil, K. M.; Balandin, A. A., Graphene-Multilayer Graphene Nanocomposites as Highly Efficient Thermal Interface Materials. Nano Lett. 2012, 12, 861-867.

(S6) Yu, A.; Ramesh, P.; Sun, X.; Bekyarova, E.; Itkis, M. E.; Haddon, R. C., Enhanced Thermal Conductivity in a Hybrid Graphite Nanoplatelet-Carbon Nanotube Filler for Epoxy Composites. $A d v$. Mater. 2008, 20, 4740-4744.

(S7) Ganguli, S.; Roy, A. K.; Anderson, D. P., Improved Thermal Conductivity for Chemically Functionalized Exfoliated Graphite/Epoxy Composites. Carbon 2008, 46, 806-817.

(S8) Kim, H. S.; Bae, H. S.; Yu, J.; Kim, S. Y., Thermal Conductivity of Polymer Composites with the Geometrical Characteristics of Graphene Nanoplatelets. Sci. Rep. 2016, 6, 1-9.

(S9) Yang, J.; Li, X.; Han, S.; Yang, R.; Min, P.; Yu, Z. Z., High-Quality Graphene Aerogels for 
Thermally Conductive Phase Change Composites with Excellent Shape Ttability. J. Mater. Chem. A 2018, 6, 5880-5886.

(S10) Yang, J.; Qi, G. Q.; Liu, Y.; Bao, R. Y.; Liu, Z. Y.; Yang, W.; Xie, B. H.; Yang, M. B., Hybrid Graphene Aerogels/Phase Change Material Composites: Thermal Conductivity, Shape-Stabilization and Light-to-Thermal Energy Storage. Carbon 2016, 100, 693-702.

(S11) Yang, J.; Li, X.; Han, S.; Zhang, Y.; Min, P.; Koratkar, N.; Yu, Z. Z., Air-Dried, High-Density Graphene Hybrid Aerogels for Phase Change Composites with Exceptional Thermal Conductivity and Shape Stability. J. Mater. Chem. A 2016, 4, 18067-18074.

(S12) Kholmanov, I.; Kim, J.; Ou, E.; Ruoff, R. S.; Shi, L., Continuous Carbon Nanotube-Ultrathin Graphite Hybrid Foams for Increased Thermal Conductivity and Suppressed Subcooling in Composite Phase Change Materials. ACS Nano 2015, 9, 11699-11707.

(S13) Dai, W.; Yu, J.; Wang, Y.; Song, Y.; Alam, F. E.; Nishimura, K.; Lin, C. T.; Jiang, N., Enhanced Thermal Conductivity for Polyimide Composites with a Three-Dimensional Silicon Carbide Nanowire@Graphene Sheets Filler. J. Mater. Chem. A 2015, 3, 4884-4891.

(S14) Ji, H.; Sellan, D. P.; Pettes, M. T.; Kong, X.; Ji, J.; Shi, L.; Ruoff, R. S., Enhanced Thermal Conductivity of Phase Change Materials with Ultrathin-Graphite Foams for Thermal Energy Storage. Energy Environ. Sci. 2014, 7, 1185-1192.

(S15) Liu, P.; Li, X.; Min, P.; Chang, X.; Shu, C.; Ding, Y.; Yu, Z. Z., 3D Lamellar-Structured Graphene Aerogels for Thermal Interface Composites with High Through-Plane Thermal Conductivity and Fracture Toughness. Nano-Micro Lett. 2021, 13, 1-15.

(S16) Li, X. H.; Liu, P.; Li, X.; An, F.; Min, P.; Liao, K. N.; Yu, Z. Z., Vertically Aligned, Ultralight and Highly Compressive All-Graphitized Graphene Aerogels for Highly Thermally Conductive 
Polymer Composites. Carbon 2018, 140, 624-633.

(S17) Lian, G.; Tuan, C. C.; Li, L.; Jiao, S.; Wang, Q.; Moon, K. S.; Cui, D.; Wong, C. P., Vertically Aligned and Interconnected Graphene Networks for High Thermal Conductivity of Epoxy Composites with Ultralow Loading. Chem. Mater. 2016, 28, 6096-6104.

(S18) Marconnet, A. M.; Yamamoto, N.; Panzer, M. A.; Wardle, B. L.; Goodson, K. E., Thermal Conduction in Aligned Carbon Nanotube-Polymer Nanocomposites with High Packing Density. ACS Nano 2011, 5, 4818-4825.

(S19) An, F.; Li, X.; Min, P.; Li, H.; Dai, Z.; Yu, Z. Z., Highly Anisotropic Graphene/Boron Nitride Hybrid Aerogels with Long-Range Ordered Architecture and Moderate Density for Highly Thermally Conductive Composites. Carbon 2018, 126, 119-127.

(S20) Hou, H.; Dai, W.; Yan, Q.; Lv, L.; Alam, F. E.; Yang, M.; Yao, Y.; Zeng, X.; Xu, J. B.; Yu, J., Graphene Size-Dependent Modulation of Graphene Frameworks Contributing to the Superior Thermal Conductivity of Epoxy Composites. J. Mater. Chem. A 2018, 6, 12091-12097.

(S21) An, F.; Li, X.; Min, P.; Liu, P.; Jiang, Z. G.; Yu, Z. Z., Vertically Aligned High-Quality Graphene Foams for Anisotropically Conductive Polymer Composites with Ultrahigh Through-Plane Thermal Conductivities. ACS Appl. Mater. Interfaces 2018, 10, 17383-17392.

(S22) Jung, H.; Yu, S.; Bae, N. S.; Cho, S. M.; Kim, R. H.; Cho, S. H.; Hwang, I.; Jeong, B.; Ryu, J. S.; Hwang, J., High Through-Plane Thermal Conduction of Graphene Nanoflake Filled Polymer Composites Melt-Processed in an L-Shape Kinked Tube. ACS Appl. Mater. Interfaces 2015, 78, $15256-15262$.

Li, Q.; Guo, Y.; Li, W.; Qiu, S.; Zhu, C.; Wei, X.; Chen, M.; Liu, C.; Liao, S.; Gong, Y.; Mishra, A. K.; Liu, L., Ultrahigh Thermal Conductivity of Assembled Aligned Multilayer 
Graphene/Epoxy Composite. Chem. Mater. 2014, 26, 4459-4465.

(S24) Wu, Z.; Xu, C.; Ma, C.; Liu, Z.; Cheng, H. M.; Ren, W., Synergistic Effect of Aligned Graphene Nanosheets in Graphene Foam for High-Performance Thermally Conductive Composites. Adv. Mater. 2019, 31, 1900199.

(S25) Shen, X.; Wang, Z.; Wu, Y.; Liu, X.; He, Y. B.; Zheng, Q.; Yang, Q. H.; Kang, F.; Kim, J. K., A Three-Dimensional Multilayer Graphene Web for Polymer Nanocomposites with Exceptional Transport Properties and Fracture Resistance. Mater. Horiz. 2018, 5, 275-284. 